\title{
TITLE:
}

\section{On asymptotic structure at null infinity in five dimensions}

\section{$\operatorname{AUTHOR}(\mathrm{S})$ :}

Tanabe, Kentaro; Tanahashi, Norihiro; Shiromizu, Tetsuya

\section{CITATION:}

Tanabe, Kentaro ... [et al]. On asymptotic structure at null infinity in five dimensions. Journal of Mathematical Physics 2010, 51(6): 062502.

ISSUE DATE:

2010-06

URL:

http://hdl.handle.net/2433/126694

RIGHT:

(C) 2010 American Institute of Physics 


\title{
On asymptotic structure at null infinity in five dimensions
}

\author{
Kentaro Tanabe, ${ }^{1, a)}$ Norihiro Tanahashi, ${ }^{2}$ and Tetsuya Shiromizu ${ }^{2}$ \\ ${ }^{1}$ Yukawa Institute for Theoretical Physics, Kyoto University, Kyoto 606-8502, Japan \\ ${ }^{2}$ Department of Physics, Kyoto University, Kyoto 606-8502, Japan
}

(Received 4 September 2009; accepted 21 April 2010; published online 3 June 2010)

We discuss the asymptotic structure of null infinity in five dimensional space-times. Since it is known that the conformal infinity is not useful for odd higher dimensions, we shall employ the coordinate based method such as the Bondi coordinate first introduced in four dimensions. Then we will define the null infinity and identify the asymptotic symmetry. We will also derive the Bondi mass expression and show its conservation law. () 2010 American Institute of Physics.

[doi:10.1063/1.3429580]

\section{INTRODUCTION}

Inspired by superstring theory, fundamental studies of higher dimensional space-time is important. One issue is the asymptotic structure at null infinity. We often introduce the conformal infinity to discuss the asymptotic structure at infinities in four dimensions. ${ }^{1,2}$ Therein the spacetime is compactified by conformal transformation and embedded into another space-time. For example, Minkowski space-time is conformally embedded into the Einstein static universe. Conformally embedded space-time has two different infinities, i.e., spatial infinity and null infinity. In higher dimensional space-times, the asymptotic structure at spatial infinity can be well-defined. The asymptotic symmetry is identified with the Poincare group and conserved quantities associated with the symmetry are constructed. ${ }^{3,4}$

On the other hand, the asymptotic structure at null infinity is not completely understood in higher dimensional space-times. ${ }^{5-7}$ Indeed, the definition of null infinity is given only in even dimensions. ${ }^{5,7}$ As seen later, the difficulty in defining null infinity as compared to spatial infinity is due to the presence of the gravitational waves at null infinity. Since there are no gravitational waves at spatial infinity, the asymptotic structure is "stationary," and the total mass and total angular momentum are conserved. On the other hand, the asymptotic structure at null infinity might be disturbed by gravitational waves. Hence, we need a stable definition of null infinity against gravitational waves. We can give such a definition in even dimensions if we use a conformal embedding method. However, we cannot do this in odd dimensional space-times because we cannot show the smoothness of the Einstein equations at null infinity. This nonsmoothness would be related to the facts that in the conformal embedding method we introduce the conformal factor $\Omega \sim 1 / r$, and the behavior of gravitational waves near null infinity is on the order of $\mathcal{O}\left(\Omega^{(d-2) / 2}\right)$ in $d$ dimensional space-times. The problem comes from the half-integer power of $\Omega$. From this smoothness only, however, we cannot show that the boundary conditions at null infinity for asymptotic flatness in the papers ${ }^{5-7}$ are the marginal and weakest conditions that allow gravitational waves at null infinity to exist. To find such marginal conditions, which the boundary conditions should be so, we must solve the Einstein equations near null infinity and clarify the freedom of gravitational waves.

In this paper, we define null infinity in five dimensional space-time. We do not use the conformal embedding method, but instead we use the Bondi coordinate to define null infinity, which was introduced first by Bondi and Sachs in four dimensions. ${ }^{8-11}$ The rest of this paper is

\footnotetext{
${ }^{a)}$ Electronic mail: tanabe@yukawa.kyoto-u.ac.jp.
} 
organized as follows. In Sec. II, we introduce the Bondi coordinate in five dimensions. In Sec. III, we define the asymptotic flatness at null infinity in five dimensions and show the robustness of the definition against gravitational waves by solving Einstein equations. In this section, we define the Bondi mass and obtain the mass loss law in five dimensions. In Sec. IV, we discuss the asymptotic symmetry at null infinity associated with the asymptotic flatness defined in Sec. III. We show that there are no supertranslations in five dimensions unlike in four dimensions. Finally, in Sec. V, we give a discussion and a summary.

\section{BONDI COORDINATE IN FIVE DIMENSIONS}

We consider five dimensional space-time. We introduce the Bondi coordinate to define asymptotic flatness at null infinity. Suppose there is a function $u\left(x^{a}\right)$ that satisfies the equation

$$
u_{, a} u_{, b} g^{a b}=0,
$$

where Latin indices run from 0 to 4 and $u_{, a}=\partial u / \partial x^{a}$. Then $u=$ constant surfaces are null hypersurfaces, and we use this function $u$ as retarded time to construct a coordinate system. Let $\theta, \phi$, and $\psi$ be angular coordinates, which are constant along gradient $u$. The periods of each of these coordinates are taken to be $\pi, 2 \pi$, and $2 \pi$, respectively. For convenience, we introduce the notation $(\theta, \phi, \psi)=x^{A}$, where capital Latin indices run from 2 to 4 . Now we define the function $r$ by the equation

$$
r^{6} \sin ^{2} \theta \cos ^{2} \theta=\operatorname{det}\left(g_{A B}\right) .
$$

Using these coordinates (we call them the Bondi coordinates)

$$
x^{0}=u, \quad x^{1}=r, \quad x^{2}=\theta, \quad x^{3}=\phi, \quad x^{4}=\psi,
$$

we can write down metrics as

$$
d s^{2}=-\left(V e^{B} / r^{2}\right) d u^{2}-2 e^{B} d u d r+r^{2} h_{A B}\left(d x^{A}+U^{A} d u\right)\left(d x^{B}+U^{B} d u\right),
$$

where

$$
h_{A B}=\left(\begin{array}{ccc}
e^{C_{1}} & \sin \theta \sinh D_{1} & \cos \theta \sinh D_{2} \\
\sin \theta \sinh D_{1} & e^{C_{2}} \sin ^{2} \theta & \sin \theta \cos \theta \sinh D_{3} \\
\cos \theta \sinh D_{2} & \sin \theta \cos \theta \sinh D_{3} & e^{C_{3} \cos ^{2} \theta}
\end{array}\right) .
$$

In the above, $V, B, h_{A B}, U^{A}, C_{1}, C_{2}, C_{3}, D_{1}, D_{2}$, and $D_{3}$ are functions of $u, r$, and $x^{A}$. In this coordinate, null infinity is represented by $r \rightarrow \infty$.

From Eq. (2), we have a relation between $C_{1}, C_{2}, C_{3}, D_{1}, D_{2}$, and $D_{3}$ as

$$
e^{C_{3}}=\frac{1+e^{C_{2}} \sinh ^{2} D_{2}+e^{C_{1}} \sinh ^{2} D_{3}-2 \sinh D_{1} \sinh D_{2} \sinh D_{3}}{e^{C_{1}+C_{2}}-\sinh ^{2} D_{1}} .
$$

As we will realize later, these five independent functions $C_{1}, C_{2}, D_{1}, D_{2}$, and $D_{3}$ correspond to the degrees of freedom of gravitational waves in five dimensional space-times.

\section{EINSTEIN EQUATION AT NULL INFINITY}

As stated in Sec. I, the definition of null infinity should not be disturbed by gravitational waves. The robustness of the null infinity definition implies that the boundary conditions imposed on the metric (4) should be compatible with Einstein equations. Hence, in order to define asymptotic structure at null infinity and show the robustness of this definition, we should specify the proper boundary conditions by solving Einstein equations in the Bondi coordinate. 


\section{A. Vacuum Einstein equations}

Since equations are very complicated, we will not write down explicit forms here. We will show the equations in a symbolic way in order to see only the essential structure of equations. See Appendix A for some details of the equations which will be used.

From $R_{r r}=0$, we have

$$
\frac{\partial B}{\partial r}=\frac{\partial \mathrm{C}^{2}}{\partial r},
$$

where C stands for $C_{1}, C_{2}, D_{1}, D_{2}$, and $D_{3}$. From $R_{r A}=0$,

$$
\frac{\partial}{\partial r}\left(r^{5} \frac{\partial U^{A}}{\partial r}\right)=r^{2}\left(\mathrm{C}+\mathrm{C}^{2}+\cdots\right)
$$

The trace and traceless parts of $R_{A B}=0$ imply that

$$
\frac{\partial}{\partial r}\left(r^{2} e^{-B} V\right)=\eta\left(\mathrm{C}, U^{A}\right)
$$

and

$$
\frac{\partial^{2}}{\partial u \partial r} \mathrm{C}=\delta\left(\mathrm{C}, U^{A}\right),
$$

where $\eta$ and $\delta$ are some functions of $\mathrm{C}$ and $U^{A}$.

Since we integrate the equations with respect to $r$ in solving the evolution equations, some integration functions $f\left(u, x^{A}\right)$ appear. Constraint equations describe the evolution of such functions.

In the Bondi coordinates, after integrating other equations, we can show that the equation $R_{u r}=0$ would be satisfied trivially.

The evolution equations $R_{u u}=0$ and $R_{u A}=0$ have the following form:

$$
\frac{\partial V}{\partial u}=r^{3}\left(\mathrm{C}^{2}+\mathrm{C}^{3}+\cdots\right)+r^{2}\left(\mathrm{C}+\mathrm{C}^{2}+\cdots\right)
$$

and

$$
r \frac{\partial U^{A}}{\partial u}=\frac{\partial \mathrm{C}}{\partial u}
$$

respectively.

If $\mathrm{C}$ is given on the initial surface $u=u_{0}$, we can determine the metric functions $B, U^{A}$, and $V$ from Eqs. (7)-(9) except for integration functions. The evolution of $\mathbb{C}$ and integration functions are described by Eqs. (10)-(12).

As seen later, the functions $\mathrm{C}=\left(C_{1}, C_{2}, D_{1}, D_{2}, D_{3}\right)$ will be identified with the freedom of gravitational waves, and Eq. (11) will govern the evolution of their total mass. Thus, to obtain a stable definition of null infinity, we should determine the asymptotic behavior of the function C. Then, using Eqs. (7)-(9), we can obtain the asymptotic behavior of $B, U^{A}$, and $V$, and the robustness against gravitational waves of these boundary conditions would be guaranteed by the evolution equations (11) and (12).

\section{B. Asymptotic behavior of gravitational fields}

From now on, we will write down all equations explicitly. When function $\mathrm{C}$ corresponds to gravitational waves, $\mathrm{C}$ behaves as $\sim 1 / r^{3 / 2}$ near null infinity. This can be seen from the solutions 
to the wave equation and/or the finiteness of the gravitational flux at null infinity. Therefore, we assume the behavior of $C_{1}, C_{2}, C_{3}, D_{1}, D_{2}$, and $D_{3}$ near null infinity such that

$$
\begin{aligned}
& C_{1}\left(u, r, x^{A}\right)=\frac{C_{11}\left(u, x^{A}\right)}{r^{3 / 2}}+O\left(1 / r^{2}\right), \\
& C_{2}\left(u, r, x^{A}\right)=\frac{C_{21}\left(u, x^{A}\right)}{r^{3 / 2}}+O\left(1 / r^{2}\right), \\
& C_{3}\left(u, r, x^{A}\right)=\frac{C_{31}\left(u, x^{A}\right)}{r^{3 / 2}}+O\left(1 / r^{2}\right), \\
& D_{1}\left(u, r, x^{A}\right)=\frac{D_{11}\left(u, x^{A}\right)}{r^{3 / 2}}+O\left(1 / r^{2}\right), \\
& D_{2}\left(u, r, x^{A}\right)=\frac{D_{21}\left(u, x^{A}\right)}{r^{3 / 2}}+O\left(1 / r^{2}\right), \\
& D_{3}\left(u, r, x^{A}\right)=\frac{D_{31}\left(u, x^{A}\right)}{r^{3 / 2}}+O\left(1 / r^{2}\right) .
\end{aligned}
$$

As noted around Eq. (6), $C_{31}$ can be written as

$$
C_{31}=-\left(C_{11}+C_{21}\right) \text {. }
$$

Then, from Eq. (7), we see that

$$
B=B_{1} / r^{3}+O\left(r^{-7 / 2}\right)
$$

near null infinity, and we obtain a relation

$$
B_{1}=-\frac{\left(C_{11}^{2}+C_{11} C_{21}+C_{21}^{2}+D_{11}^{2}+D_{21}^{2}+D_{31}^{2}\right)}{8} .
$$

Furthermore, integrating Eq. (8), we have the relations

$$
\begin{gathered}
U_{\theta 1}=\frac{2\left(C_{11} \cot \theta-C_{21} \cot \theta-2 C_{11} \tan \theta-C_{21} \tan \theta+C_{11, \theta}+D_{11, \phi} \csc \theta+D_{21, \psi} \sec \theta\right)}{5}, \\
U_{\phi 1} \sin \theta=\frac{2\left(2 D_{11} \cot \theta-D_{11} \tan \theta+D_{11, \theta}+C_{21, \phi} \csc \theta+D_{31, \psi} \sec \theta\right)}{5},
\end{gathered}
$$

and

$$
U_{\psi 1} \cos \theta=\frac{2\left(D_{21} \cot \theta-2 D_{21} \tan \theta+D_{21, \theta}+D_{31, \phi} \csc \theta-\left(C_{11, \psi}+C_{21, \psi}\right) \sec \theta\right)}{5},
$$

where $U_{A 1}$ is the coefficient of the expansion defined as 


$$
U^{A}=\frac{U_{A 1}}{r^{5 / 2}}+O\left(1 / r^{3}\right)
$$

Then, from Eq. (9), we find

$$
V=r^{2}+V_{1}\left(u, x^{A}\right) r^{1 / 2}-M\left(u, x^{A}\right)+O\left(r^{-1 / 2}\right)
$$

where

$$
V_{1}=-\frac{2}{3}\left(\frac{1}{\sin \theta \cos \theta}\left(U_{\theta 1} \sin \theta \cos \theta\right)_{, \theta}+U_{\phi 1, \phi}+U_{\psi 1, \psi}\right)
$$

and $M\left(u, x^{A}\right)$ is an integration constant.

In Eq. (10), $C_{11, u}, C_{21, u}, D_{11, u}, D_{21, u}$, and $D_{31, u}$ do not appear and then this means that we can fix them freely as initial conditions. This freedom corresponds to the degrees of freedom of gravitational waves in five dimensions.

Finally, from Eq. (11), we obtain the following formula:

$$
\begin{aligned}
\frac{\partial M\left(u, x^{A}\right)}{\partial u} & =-\frac{1}{3}\left(\left(C_{11, u}\right)^{2}+C_{11, u} C_{21, u}+\left(C_{21, u}\right)^{2}+\left(D_{11, u}\right)^{2}+\left(D_{21, u}\right)^{2}+\left(D_{31, u}\right)^{2}\right) \\
& =-\frac{1}{3}\left(\left(C_{11, u}+C_{21, u} / 2\right)^{2}+3\left(C_{21}\right)^{2} / 4+\left(D_{11, u}\right)^{2}+\left(D_{21, u}\right)^{2}+\left(D_{31, u}\right)^{2}\right) .
\end{aligned}
$$

Equation (28) represents mass loss rate by gravitational waves, and the total mass always decreases as in four dimensions. Then $M\left(u, x^{A}\right)$ describes the mass in $u=$ constant surfaces.

\section{Boundary conditions}

As shown in Sec. III B, in the presence of gravitational waves, asymptotic behaviors of metric (4) in leading order should be

$$
\begin{gathered}
V=r^{2}+V_{1}\left(u, x^{A}\right) r^{1 / 2}-M\left(u, x^{A}\right)+O\left(1 / r^{1 / 2}\right), \\
B=\frac{B_{1}\left(u, x^{A}\right)}{r^{3}}+O\left(1 / r^{7 / 2}\right), \\
U^{A}=\frac{U_{A 1}\left(u, x^{A}\right)}{r^{5 / 2}}+O\left(1 / r^{3}\right), \\
\mathrm{C}=\frac{\mathrm{C}_{1}\left(u, x^{A}\right)}{r^{3 / 2}}+O\left(1 / r^{2}\right) .
\end{gathered}
$$

In four dimensions, boundary conditions at null infinity for asymptotic flatness are determined by this leading behavior. As shown below, however, in dimensions higher than 4, further conditions are needed for asymptotic flatness.

We consider asymptotic behavior in next-to-leading order terms. The function C can be expanded as follows:

$$
\mathrm{C}=\frac{\mathrm{C}_{1}\left(u, x^{A}\right)}{r^{3 / 2}}+\frac{\mathrm{A}\left(u, x^{A}\right)}{r^{2}}+\frac{\mathrm{C}_{2}\left(u, x^{A}\right)}{r^{5 / 2}}+\frac{\mathrm{C}_{3}\left(u, x^{A}\right)}{r^{3}}+O\left(1 / r^{7 / 2}\right) .
$$

The equation $R_{A B}=0$, which describes the evolution of $\mathrm{C}$, becomes 


$$
\frac{\mathrm{A}_{, u}}{r}+O\left(r^{-3 / 2}\right)=0
$$

and then we see that

$$
\frac{\partial \mathrm{A}}{\partial u}=0
$$

At spatial infinity, asymptotic flatness requires that the Weyl tensor on spatial infinity behaves like $\sim r^{-5}$ in order for the Taub-NUT charge to vanish. ${ }^{4}$ This implies that A vanishes at spatial infinity $(u=-\infty)$. From Eq. (35), A should vanish for asymptotic flatness at null infinity

$$
A=0 .
$$

Then, from Eqs. (7)-(9), we can show that other metric functions can be expanded as follows:

$$
\begin{gathered}
V=r^{2}+V_{1}\left(u, x^{A}\right) r^{1 / 2}-M\left(u, x^{A}\right)+O\left(1 / r^{1 / 2}\right), \\
B=\frac{B_{1}\left(u, x^{A}\right)}{r^{3}}+\frac{B_{2}\left(u, x^{A}\right)}{r^{4}}+\frac{B_{3}\left(u, x^{A}\right)}{r^{9 / 2}}+O\left(1 / r^{5}\right), \\
U^{A}=\frac{U_{A 1}\left(u, x^{A}\right)}{r^{5 / 2}}+\frac{U_{A 2}\left(u, x^{A}\right)}{r^{7 / 2}}+\frac{U_{A 3}\left(u, x^{A}\right)}{r^{4}}+O\left(1 / r^{9 / 2}\right) .
\end{gathered}
$$

(The $U_{A 3}$ term corresponds to the angular momentum. This can be seen from the comparison with the asymptotic behavior of Myers-Perry solutions. ${ }^{12}$ ) Thus, boundary conditions at null infinity for asymptotic flatness are

$$
\mathrm{C}=\frac{\mathrm{C}_{1}\left(u, x^{A}\right)}{r^{3 / 2}}+\frac{\mathrm{C}_{2}\left(u, x^{A}\right)}{r^{5 / 2}}+\frac{\mathrm{C}_{3}\left(u, x^{A}\right)}{r^{3}}+O\left(1 / r^{7 / 2}\right),
$$

and Eqs. (37)-(39).

\section{ASYMPTOTIC SYMMETRY AT NULL INFINITY}

In this section, we consider asymptotic symmetry at null infinity. Asymptotic symmetry should be defined as transformations preserving the boundary conditions (37)-(40). By infinitesimal transformation $\xi$, the metric is transformed as

$$
\delta g_{a b}=2 \nabla_{(a} \xi_{b)} .
$$

To preserve the boundary conditions given in Sec. III, the variation of metric $\delta g_{a b}$ should satisfy

$$
\begin{gathered}
\delta g_{r r}=0, \quad \delta g_{r A}=0, \quad g^{A B} \delta g_{A B}=0, \\
\delta g_{u u}=O\left(r^{-3 / 2}\right), \quad \delta g_{u r}=O\left(r^{-3}\right), \quad \delta g_{u A}=O\left(r^{-1 / 2}\right), \quad \delta g_{A B}=O\left(r^{1 / 2}\right) .
\end{gathered}
$$

Here, as a first step, we will consider leading order terms.

From Eq. (42), we see that the components of infinitesimal transformation $\xi$ must take the following forms:

$$
\begin{gathered}
\xi_{r}=f\left(u, x^{A}\right) e^{B}, \\
\xi_{B} g^{A B}=f^{A}\left(u, x^{A}\right)-f U^{A}+\int_{r}^{\infty} d r^{\prime} e^{B} f_{, B} g^{A B},
\end{gathered}
$$




$$
\xi_{u}=-\frac{r e^{B}}{3}\left(-\xi_{A, B}+\xi_{C} \Gamma_{A B}^{C}+\xi_{r} \Gamma_{A B}^{r}\right) g^{A B} .
$$

The infinitesimal transformation $\xi$ has four free parameters $f, f^{A} . f$ and $f^{A}$ corresponds to translations and Lorentz transformation, respectively. The other components of metric variation become

$$
\begin{gathered}
\delta g_{u u}=\frac{2 r}{3} \mathcal{D}_{A} f_{, u}^{A}+\frac{2}{3}\left(3 f+\mathcal{D}^{2} f\right)_{, u}+\frac{2}{r^{1 / 2}} h_{A B}^{(0)} f_{, u}^{A} U_{B 1}+O\left(r^{-3 / 2}\right) \\
\delta g_{u r}=\frac{1}{3}\left(\mathcal{D}_{A} f^{A}+3 f_{, u}\right)+\frac{1}{5 r^{5 / 2}} h^{(1) A B} \mathcal{D}_{A} \mathcal{D}_{B} f+O\left(r^{-3}\right) \\
\delta g_{u A}=r^{2} h_{A B}^{(0)} f_{, u}^{B}+\frac{r}{3} \mathcal{D}_{A}\left(3 f_{, u}+\mathcal{D}_{B} f^{B}\right)+r^{1 / 2} h_{A B}^{(1)} f_{, u}^{B}+\frac{1}{3} \mathcal{D}_{A}\left(\mathcal{D}^{2} f+3 f\right)+O\left(r^{-1 / 2}\right) \\
\delta g_{A B}=\frac{2 r^{2}}{3}\left(-\mathcal{D}_{C} f^{C} h_{A B}^{(0)}+3 \mathcal{D}_{(A} f_{B)}\right)+\frac{2 r}{3}\left(-\mathcal{D}^{2} f h_{A B}^{(0)}+3 \mathcal{D}_{A} \mathcal{D}_{B} f\right)+T_{A B}\left(u, x^{A}\right) r^{1 / 2}+O\left(r^{-1 / 2}\right)
\end{gathered}
$$

where $X_{(A B)}:=(1 / 2)\left(X_{A B}+X_{B A}\right)$ for some tensor $X_{A B}$ and $T_{A B}$ is some traceless tensor with respect to $h_{A B}^{(0)}$. In the above, we expanded the metric $h_{A B}$ as

$$
h_{A B}=h_{A B}^{(0)}+\frac{1}{r^{3 / 2}} h_{A B}^{(1)}+O\left(r^{-5 / 2}\right)
$$

and $h^{(1) A B}=h^{(0) A C} h^{(0) B D} h_{C D}^{(1)}$. In the Bondi coordinate, $h_{A B}^{(0)}$ and $h_{A B}^{(1)}$ are

$$
h_{A B}^{(0)}=\left(\begin{array}{ccc}
1 & 0 & 0 \\
0 & \sin ^{2} \theta & 0 \\
0 & 0 & \cos ^{2} \theta
\end{array}\right)
$$

and

$$
h_{A B}^{(1)}=\left(\begin{array}{ccc}
C_{11} & D_{11} \sin \theta & D_{21} \cos \theta \\
D_{11} \sin \theta & C_{21} \sin ^{2} \theta & D_{31} \sin \theta \cos \theta \\
D_{21} \cos \theta & D_{31} \sin \theta \cos \theta & -\left(C_{11}+C_{21}\right) \cos ^{2} \theta
\end{array}\right) .
$$

Note that $h_{A B}^{(1)}$ is traceless, $h^{(0) A B} h_{A B}^{(1)}=0$.

To satisfy the condition (43), we find that $f^{A}$ and $f$ should satisfy the following equations:

$$
\begin{gathered}
f^{A}{ }_{, u}=0, \\
\mathcal{D}_{A} f_{B}+\mathcal{D}_{B} f_{A}=-2 \frac{\partial f}{\partial u} h_{A B}^{(0)}, \\
\mathcal{D}_{A} \mathcal{D}_{B} f=\frac{1}{3} \mathcal{D}^{2} f h_{A B}^{(0)} .
\end{gathered}
$$

Integrating the trace part of Eq. (55) under the condition (54), we obtain 


$$
f=-\frac{u}{3} F+\alpha\left(x^{A}\right)
$$

where $F:=\mathcal{D}_{A} f^{A}$ and $\alpha\left(x^{A}\right)$ is an arbitrary function of $x^{A}$. Here, we can show from Eq. (55) that $F$ satisfies

$$
\mathcal{D}_{A} \mathcal{D}_{B} F=\frac{1}{3} \mathcal{D}^{2} F h_{A B}^{(0)}
$$

and

$$
\mathcal{D}^{2} F+3 F=0
$$

See Appendix B for the derivation. The general solution to this is given by

$$
F=E_{x} \sin \theta \cos \phi+E_{y} \sin \theta \sin \phi+E_{z} \cos \theta \cos \psi+E_{w} \cos \theta \sin \psi,
$$

where $E_{x}, E_{y}, E_{z}$, and $E_{w}$ are constants. Then, from Eqs. (56) and (57), we see that

$$
\mathcal{D}_{A} \mathcal{D}_{B} \alpha=\frac{1}{3} \mathcal{D}^{2} \alpha h_{A B}^{(0)}
$$

holds. The solution to this is

$$
\alpha=a_{u}+a_{x} \sin \theta \cos \phi+a_{y} \sin \theta \sin \phi+a_{z} \cos \theta \cos \psi+a_{w} \cos \theta \sin \psi,
$$

where $a_{u}, a_{x}, a_{y}, a_{z}$, and $a_{w}$ are constants. As a summary, the general solution for $f$ is given by

$$
f=e_{u}+e_{x}(u) \sin \theta \cos \phi+e_{y}(u) \sin \theta \sin \phi+e_{z}(u) \cos \theta \cos \psi+e_{w}(u) \cos \theta \sin \psi,
$$

where $e_{u}:=a_{u}$ is constant, $e_{x}(u):=-(u / 3) E_{x}+a_{x}, e_{y}(u):=-(u / 3) E_{y}+a_{y}, e_{z}(u):=-(u / 3) E_{z}+a_{z}$, and $e_{w}(u):=-(u / 3) E_{w}+a_{w}$. Now we can show that

$$
\mathcal{D}_{A}\left(\mathcal{D}^{2} f+3 f\right)=0, \quad \partial_{u}\left(\mathcal{D}^{2} f+3 f\right)=0
$$

hold. Using the above equations, at a glance, we see that Eq. (43) becomes

$$
\begin{gathered}
\delta g_{u u}=O\left(r^{-3 / 2}\right), \\
\delta g_{u r}=\frac{1}{5 r^{5 / 2}} h^{(1) A B} \mathcal{D}_{A} \mathcal{D}_{B} f+O\left(r^{-3}\right), \\
\delta g_{u A}=O\left(r^{-1 / 2}\right), \\
\delta g_{A B}=\frac{2 r^{2}}{3}\left(-\mathcal{D}_{C} f^{C} h_{A B}^{(0)}+3 \mathcal{D}_{(A} f_{B)}\right)+T_{A B}\left(u, x^{A}\right) r^{1 / 2}+O\left(r^{-1 / 2}\right) .
\end{gathered}
$$

$h^{(1) A B} \mathcal{D}_{A} \mathcal{D}_{B} f$ in Eq. (66) vanishes because of $\mathcal{D}_{A} \mathcal{D}_{B} f \propto h_{A B}^{(0)}$ and the trace-free property of $h^{(1) A B}$. Noting that the condition of (55) is rearranged as $\mathcal{D}_{A} f_{B}+\mathcal{D}_{B} f_{A}=(2 / 3) \mathcal{D}^{C} f_{C} h_{A B}^{(0)}$, we can show that $\delta g_{A B}=O\left(r^{1 / 2}\right)$. As a consequence, the transformation satisfying the conditions of Eqs. (54)-(56) does not disturb the boundary condition of Eqs. (37)-(40) at leading order. By a straightforward calculation, it can be shown that these transformations keep the metric satisfying the boundary conditions at next-to-leading order.

Thus, asymptotic symmetry is generated by $f$ and $f^{A}$ satisfying Eqs. (54)-(56). The part of $f$ not proportional to $u$ generates a translation group. Since this part has only five degrees of freedom, there is no supertranslation freedom unlike in four dimensions. (The fact that supertranslation freedom does not exist in higher dimensions is first pointed out in Ref. 5 by using conformal method in even dimensions. We show that in five dimensions using the Bondi coordinates.) $f^{A}$ 
generates a Lorentz transformation group, so asymptotic symmetry at null infinity in five dimensional space-time is a Poincaré group, which is semidirect of a five dimensional transformation group and Lorentz group.

\section{SUMMARY AND DISCUSSION}

In this paper, we define asymptotic flatness at null infinity in five dimensional space-time by using the Bondi coordinates. In the conformal embedding method, we cannot show the smoothness of the asymptotic structure at null infinity because the gravitational waves behave like $\Omega^{3 / 2}$ $\sim r^{-3 / 2}$ near null infinity and the regularity of gravitational fields at null infinity is not guaranteed, in general, in five dimensions. On the other hand, in the Bondi coordinates, we can show the robustness of the asymptotic structure at null infinity, which is defined by boundary conditions of Eqs. (37)-(40). Solving Einstein equations under these boundary conditions, we find that total mass always decreases by gravitational waves as in four dimensions. In addition, we show that the asymptotic symmetry at null infinity would be a Poincaré group in five dimensions.

In four dimensions, asymptotic symmetry at null infinity is not a Poincare group. There are the so-called supertranslation freedoms, i.e., there are infinite dimensional translations. This supertranslation freedom comes from the freedom of the Bondi coordinates. In the Bondi coordinates, coordinate transformation is described by the parameters $f$ and $f^{A}$ in Eqs. (44)-(46), which correspond to translation and Lorentz transformation, respectively. If $f^{A}=0$ (pure translation), we may expect that $f$ has only four independent solutions. In general, however, there are conditions that $f$ should be a functions on a 2 -sphere and then $f$ has functional freedom, i.e., infinite dimensional degrees of freedom. This infinite dimensional set, which has an Abelian group structure, is called a supertranslational group.

In four dimensional Minkowski space-time without any physical perturbations, the term $O(r)$ of $\delta g_{A B}$ should vanish, and this condition reduces infinite dimensional supertranslation to four dimensional translation. In general, however, since gravitational waves contribute to $g_{A B}$ with $O(r)$ terms, which is the same order with metric variance, we cannot reduce supertranslation to translation. That is, we cannot distinguish the supertranslational ambiguity from gravitational waves. Thus, the asymptotic symmetry at null infinity in four dimensions is not a Poincaré group.

On the other hand, as shown in this paper, in five dimensions, there is no supertranslational freedom. This is because the behavior of gravitational waves in five dimensions is $1 / r^{3 / 2}$ and this contributes to $g_{A B}$ with $O\left(r^{1 / 2}\right)$ terms. In asymptotically flat five dimensional space-time, the term of $O(r)$ in $\delta g_{A B}$, which could be a contribution from the supertranslation, should vanish to maintain asymptotic flatness. This condition eliminates supertranslational freedom and makes the asymptotic symmetry a Poincaré group. Although we have only shown this feature in five dimensions, we expect that this feature would be common to the higher dimensional space-time, in general, because gravitational waves in $d>4$ dimensions decay $1 / r^{(d-2) / 2}$ near null infinity faster than supertranslation $O(1 / r)$.

In four dimensions, since there are supertranslations, we cannot choose the preferred rotational axis. The definition of angular momentum at null infinity does not have a precise meaning. Supertranslations and gravitational waves both contribute to angular momentum change, and we cannot distinguish one from the other. However, since there is no supertranslation in five dimensions, we can define the total angular momentum at null infinity and observe the change of the total angular momentum by gravitational waves. Furthermore, as we show the robustness of null infinity definition in five dimensions using the Bondi coordinates, it may be possible for us to redefine asymptotic flatness at null infinity using a conformal embedding method covariantly. This is left for future issue.

Another remaining issue is the extension of our work to dimensions higher than 7. Using the Bondi coordinates, we have to go on step by step. It will be nice to have a systematic analysis for that. This is also our future work. 


\section{ACKNOWLEDGMENTS}

K.T. was supported by JSPS Grant-in-Aid for Scientific Research. N.T. and T.S. were partially supported by Grant-in-Aid for Scientific Research from Ministry of Education, Science, Sports and Culture of Japan (Grant Nos. 2056381, 20540258, 21111006, and 19GS0219) and the JapanU.K. Research Cooperative Programs. This work was also supported by the Grant-in-Aid for the Global COE Program "The Next Generation of Physics, Spun from Universality and Emergence" from the Ministry of Education, Culture, Sports, Science and Technology (MEXT) of Japan.

\section{APPENDIX A: EINSTEIN EQUATION}

We write the components of an Einstein equation explicitly in the expansion form with $1 / r$. If for example, we expand function $C_{1}\left(u, r, x^{A}\right)$ such that

$$
C_{1}=\frac{C_{11}\left(u, x^{A}\right)}{r \sqrt{r}}+\frac{A\left(u, x^{A}\right)}{r^{2}}+O\left(r^{-5 / 2}\right) .
$$

The Einstein equation $R_{\theta \theta}=0$ becomes

$$
-\frac{A_{, u}}{2 r}+O\left(r^{-3 / 2}\right)=0 .
$$

That is, $\partial A\left(u, x^{A}\right) / \partial u=0$. In Ref. 4 , it was shown that from asymptotic flatness at spatial infinity, $\left.A\left(u, x^{A}\right)\right|_{u=-\infty}=0$. From this fact and $\partial A\left(u, x^{A}\right) / \partial u=0$, we find that $A\left(u, x^{A}\right)=0$ on null infinity. We can show in the same way that the $O\left(r^{-2}\right)$ term in $C_{2}, D_{1}, D_{2}$, and $D_{3}$ should also vanish.

Thus, function $\mathrm{C}$ is expanded as follows:

$$
\begin{aligned}
& C_{1}\left(u, r, x^{A}\right)=\frac{C_{11}\left(u, x^{A}\right)}{r \sqrt{r}}+\frac{C_{12}\left(u, x^{A}\right)}{r^{2} \sqrt{r}}+\frac{C_{13}\left(u, x^{A}\right)}{r^{3}}+O\left(1 / r^{7 / 2}\right), \\
& C_{2}\left(u, r, x^{A}\right)=\frac{C_{21}\left(u, x^{A}\right)}{r \sqrt{r}}+\frac{C_{22}\left(u, x^{A}\right)}{r^{2} \sqrt{r}}+\frac{C_{23}\left(u, x^{A}\right)}{r^{3}}+O\left(1 / r^{7 / 2}\right), \\
& D_{1}\left(u, r, x^{A}\right)=\frac{D_{11}\left(u, x^{A}\right)}{r \sqrt{r}}+\frac{D_{12}\left(u, x^{A}\right)}{r^{2} \sqrt{r}}+\frac{D_{13}\left(u, x^{A}\right)}{r^{3}}+O\left(1 / r^{7 / 2}\right), \\
& D_{2}\left(u, r, x^{A}\right)=\frac{D_{21}\left(u, x^{A}\right)}{r \sqrt{r}}+\frac{D_{22}\left(u, x^{A}\right)}{r^{2} \sqrt{r}}+\frac{D_{23}\left(u, x^{A}\right)}{r^{3}}+O\left(1 / r^{7 / 2}\right), \\
& D_{3}\left(u, r, x^{A}\right)=\frac{D_{31}\left(u, x^{A}\right)}{r \sqrt{r}}+\frac{D_{32}\left(u, x^{A}\right)}{r^{2} \sqrt{r}}+\frac{D_{33}\left(u, x^{A}\right)}{r^{3}}+O\left(1 / r^{7 / 2}\right) .
\end{aligned}
$$

Then we find from the Einstein equation that the other metric function in (4) should be expanded as follows:

$$
\begin{gathered}
V=r^{2}+V_{1}\left(u, x^{A}\right) \sqrt{r}+M\left(u, x^{A}\right)+O\left(1 / r^{1 / 2}\right), \\
B=\frac{B_{1}\left(u, x^{A}\right)}{r^{3}}+\frac{B_{2}\left(u, x^{A}\right)}{r^{4}}+O\left(1 / r^{9 / 2}\right),
\end{gathered}
$$




$$
U^{A}=\frac{U_{A 1}\left(u, x^{A}\right)}{r^{5 / 2}}+\frac{U_{A 2}\left(u, x^{A}\right)}{r^{7 / 2}}+\frac{U_{A 3}\left(u, x^{A}\right)}{r^{4}}+O\left(1 / r^{9 / 2}\right) .
$$

Now we can write down each component of the Einstein equation as follows.

$$
R_{r r}=0 \text { : }
$$

$$
\begin{aligned}
& -\frac{9}{8 r^{5}}\left(8 B_{1}+C_{11}^{2}+C_{11} C_{21}+C_{21}^{2}+D_{11}^{2}+D_{21}^{2}+D_{31}^{2}\right)-\frac{3}{8 r^{6}}\left(32 B_{2}+10 C_{11} C_{12}+5 C_{12} C_{21}+5 C_{11} C_{22}\right. \\
& \left.\quad+10 C_{21} C_{22}+10 D_{11} D_{12}+10 D_{21} D_{22}+10 D_{31} D_{32}\right)+O\left(r^{-13 / 2}\right)=0 .
\end{aligned}
$$

$R_{r \theta}=0$ :

$-\frac{3}{8 r^{5 / 2}}\left(2 C_{11} \cot \theta-2 C_{21} \cot \theta-4 C_{11} \tan \theta-2 C_{21} \tan \theta-5 U_{\theta 1}+2 D_{21, \psi} \sec \theta+2 D_{11, \phi} \csc \theta\right.$

$\left.+2 C_{11, \theta}\right)+\frac{1}{8 r^{7 / 2}}\left(-10 C_{12} \cot \theta+10 C_{22} \cot \theta+20 C_{12} \tan \theta+10 C_{22} \tan \theta+7 U_{\theta 2}\right.$

$\left.-10 D_{22, \psi} \sec \theta-10 D_{12, \phi} \csc \theta-10 C_{12, \theta}\right)+O\left(r^{-4}\right)=0$.

$R_{r \phi}=0$ :

$-\frac{3}{8 r^{5 / 2}}\left(4 D_{11} \cos \theta-2 D_{11} \sin \theta \tan \theta-5 U_{\phi 1} \sin ^{2} \theta+2 D_{31, \psi} \tan \theta+2 C_{21, \phi}+2 D_{11, \theta} \sin \theta\right)$

$+\frac{1}{8 r^{7 / 2}}\left(-20 D_{12} \cos \theta+10 D_{12} \sin \theta \tan \theta+7 U_{\phi 2} \sin ^{2} \theta-10 D_{12, \theta} \sin \theta-10 C_{22, \phi}\right.$

$\left.-10 D_{32, \psi} \tan \theta\right)+O\left(r^{-4}\right)=0$.

$R_{r \psi}=0$ :

$\frac{3}{8 r^{5 / 2}}\left(-2 D_{21} \cot \theta \cos \theta+4 D_{21} \sin \theta+5 U_{\psi 1} \cos ^{2} \theta+2 C_{11, \psi}+2 C_{21, \psi}-2 D_{31, \phi} \cot \theta\right.$

$\left.-2 D_{21, \theta} \cos \theta\right)+\frac{1}{8 r^{7 / 2}}\left(-10 D_{22} \cos \theta \cot \theta+20 D_{22} \sin \theta+7 U_{\psi 2} \cos ^{2} \theta+10 C_{12, \psi}+10 C_{22, \psi}\right.$

$\left.-10 D_{32, \phi} \cot \theta-10 D_{22, \theta} \cos \theta\right)+O\left(r^{-9 / 2}\right)=0$.

$R_{\theta \theta}=0$ :

$\frac{1}{8 r^{3 / 2}}\left(-13 C_{11}-8 U_{\theta 1} \cot \theta+8 U_{\theta 1} \tan \theta-4 V_{1}-8 D_{21, \psi} \sec \theta \tan \theta-8 U_{\psi 1, \psi}-8 U_{\phi 1, \phi}\right.$

$-4 C_{11, \phi \phi} \csc ^{2} \theta-4 C_{11, \psi \psi} \sec ^{2} \theta+8 D_{11, \phi} \cot \theta \csc \theta+4 C_{11, \theta} \cot \theta-12 C_{11, \theta} \tan \theta$

$\left.-8 C_{21, \theta} \tan \theta-12 U_{\theta 1, \theta}+8 D_{21, \theta \psi} \sec \theta+8 D_{11, \theta \phi} \csc \theta+4 C_{11, \theta \theta}-8 C_{21, \theta} \cot \theta-8 C_{12, u}\right)$

$+\frac{3}{2 r^{2}}\left(C_{13, u}-D_{11} D_{11, u}-D_{21} D_{21, u}\right)+O\left(r^{-5 / 2}\right)=0$.

$R_{\theta \phi}=0:$ 
$\frac{1}{8 r^{3 / 2}}\left(3 D_{11} \sin \theta-4 D_{31, \psi}-4 D_{31, \psi} \tan ^{2} \theta-4 D_{11, \psi \psi} \sec \theta \tan \theta-4 C_{11, \phi} \cot \theta-8 C_{11, \phi} \tan \theta\right.$

$-4 C_{21, \phi} \cot \theta-4 C_{21, \phi} \tan \theta-2 U_{\theta 1, \phi}+4 D_{21, \phi \psi} \sec \theta-2 U_{\phi 1, \theta} \sin ^{2} \theta+4 D_{31, \theta \psi} \tan \theta$

$\left.+4 C_{11, \theta \phi}+4 C_{21, \theta \phi}-8 D_{12, u} \sin \theta\right)+\frac{3 \sin \theta}{4 r^{2}}\left(D_{11} C_{11, u}+D_{11} C_{21, u}+C_{11} D_{11, u}+C_{21} D_{11, u}\right.$

$\left.-2 D_{13, u}+D_{31} D_{21, u}+D_{21} D_{31, u}\right)+O\left(r^{-5 / 2}\right)=0$.

$R_{\theta \psi}=0$ :

$\frac{1}{8 r^{3 / 2}}\left(3 D_{21} \cos \theta+4 C_{11, \psi} \cot \theta-4 C_{21, \psi} \cot \theta-4 C_{21, \psi} \tan \theta-2 U_{\theta 1, \psi}+4 D_{31, \phi}+4 D_{31, \phi} \cot ^{2} \theta\right.$

$+4 D_{11, \phi \psi} \csc \theta-4 D_{21, \phi \phi} \cot \theta \csc \theta-2 U_{\psi 1, \theta} \cos ^{2} \theta-4 C_{21, \theta \psi}+4 D_{31, \theta \phi} \cot \theta$

$\left.-8 D_{22, u} \cos \theta\right)+\frac{3 \cos \theta}{4 r^{2}}\left(D_{21} C_{21, u}-D_{31} D_{11, u}+C_{21} D_{21, u}+D_{23, u}-D_{11} D_{31, u}\right)+O\left(r^{-5 / 2}\right)=0$.

$R_{\phi \phi}=0:$

$\frac{1}{8 r^{3 / 2}}\left(-16 C_{11} \sin ^{2} \theta+3 C_{21} \sin ^{2} \theta-12 U_{\theta 1} \cos \theta \sin \theta+8 U_{\theta 1} \sin ^{2} \theta \tan \theta-4 V_{1} \sin ^{2} \theta\right.$

$+8 D_{21, \psi} \sin \theta-8 U_{\psi 1, \psi} \sin ^{2} \theta-4 C_{21, \psi \psi} \tan ^{2} \theta+8 D_{11, \phi} \cos \theta-8 D_{11, \phi} \sin \theta \tan \theta$

$-12 U_{\phi 1, \phi} \sin ^{2} \theta+8 D_{31, \phi \psi} \tan \theta+4 C_{21, \phi \phi}+8 C_{11, \theta} \cos \theta \sin \theta-4 C_{21, \theta} \cos \theta \sin \theta$

$\left.+4 C_{21, \theta} \sin ^{2} \theta \tan \theta-8 U_{\theta 1, \theta} \sin ^{2} \theta+8 D_{11, \theta \phi} \sin \theta-4 C_{21, \theta \theta} \sin ^{2} \theta-8 C_{22, u} \sin ^{2} \theta\right)$

$-\frac{3 \sin ^{2} \theta}{2 r^{2}}\left(C_{23, u}-D_{11} D_{11, u}-D_{31} D_{31, u}\right)+O\left(r^{-5 / 2}\right)=0$.

$R_{\phi \psi}=0$ :

$\frac{1}{8 r^{3 / 2}}\left(4 D_{31} \cos ^{2} \theta \cot \theta+11 D_{31} \cos \theta \sin \theta+4 D_{31} \sin ^{2} \theta \tan \theta+8 D_{11, \psi} \cos \theta+4 D_{11, \psi} \sin \theta \tan \theta\right.$

$-2 U_{\phi 1, \psi} \sin ^{2} \theta-4 D_{21, \phi} \cos \theta \cot \theta-8 D_{21, \phi} \sin \theta-2 U_{\psi 1, \phi} \cos ^{2} \theta-4 C_{11, \phi \psi}-4 D_{31, \theta} \cos ^{2} \theta$

$\left.+4 D_{31, \theta} \sin ^{2} \theta+4 D_{11, \theta \psi} \sin \theta+4 D_{21, \theta \phi} \cos \theta-D_{31, \theta \theta} \cos \theta \sin \theta-8 D_{32, u} \cos \theta \sin \theta\right)$

$-\frac{3 \cos \theta \sin \theta}{4 r^{2}}\left(D_{31} C_{11, u}-D_{21} D_{11, u}-D_{11} D_{21, u}+C_{11} D_{31, u}+D_{33, u}\right)+O\left(r^{-5 / 2}\right)=0$.

$R_{\psi \psi}=0$ :

$\frac{1}{8 r^{3 / 2}}\left(-19 C_{11} \cos ^{2} \theta-3 C_{21} \cos ^{2} \theta-8 U_{\theta 1} \cos ^{2} \theta \cot \theta-4 V_{1} \cos ^{2} \theta+12 U_{\theta 1} \cos \theta \sin \theta\right.$

$+8 D_{21, \psi} \cos \theta \cot \theta-8 D_{21, \psi} \sin \theta-12 U_{\psi 1, \psi} \cos ^{2} \theta-4 C_{11, \psi \psi}-4 C_{21, \psi \psi}-8 D_{11, \phi} \cos \theta$

$-8 U_{\phi 1, \phi} \cos ^{2} \theta+8 D_{31, \phi \psi} \cot \theta+4 C_{11, \phi \phi} \cot ^{2} \theta+4 C_{21, \phi \phi} \cot ^{2} \theta+4 C_{11, \theta} \cos ^{2} \theta \cot \theta$

$-12 C_{11, \theta} \cos \theta \sin \theta+4 C_{21, \theta} \cos ^{2} \theta \cot \theta-4 C_{21, \theta} \cos \theta \sin \theta-8 U_{\theta 1, \theta} \cos ^{2} \theta$

$\left.+8 D_{21, \theta \psi} \cos \theta+8 C_{11, \theta \theta} \cos ^{2} \theta+8\left(C_{12, u}+C_{22, u}\right) \cos ^{2} \theta\right)+\frac{3 \cos ^{2} \theta}{2 r^{2}}\left(C_{13, u}+C_{23, u}-2 D_{11} D_{11, u}\right.$ 


$$
\left.-D_{21} D_{21, u}-D_{31} D_{31, u}\right)+O\left(r^{-5 / 2}\right)=0 .
$$

$R_{u u}=0$ :

$$
\begin{aligned}
& \frac{1}{2 r^{5 / 2}}\left(3 V_{1, u}+2(\cot \theta-\tan \theta) U_{\theta 1, u}+2 U_{\theta 1, u \theta}+2 U_{\phi 1, u \phi}+2 U_{\psi 1, u \psi}\right)-\frac{1}{2 r^{3}}\left(3 M_{, u}-\left(C_{11, u}\right)^{2}\right. \\
& \left.-C_{11, u} C_{21, u}-\left(C_{21, u}\right)^{2}-\left(D_{11, u}\right)^{2}-\left(D_{21, u}\right)^{2}-\left(D_{31, u}\right)^{2}\right)+O\left(r^{-7 / 2}\right)=0 .
\end{aligned}
$$

$R_{u \theta}=0:$

$-\frac{1}{4 r^{3 / 2}}\left(2 C_{11, u} \cot \theta-4 C_{11, u} \tan \theta+2 C_{21, u} \cot \theta-2 C_{21, u} \tan \theta-5 U_{\theta 1, u}+D_{21, u \psi} \sec \theta D_{11, u \phi} \csc \theta\right.$

$$
\left.+2 C_{11, u \theta}\right)+\frac{1}{8 r^{5 / 2}}\left(-U_{\theta 1}-8 U_{\psi 1, \psi} \tan \theta-4 U_{\theta 1, \psi \psi} \sec ^{2} \theta+8 U_{\phi 1, \phi} \cot \theta-4 U_{\theta 1, \phi \phi} \csc ^{2} \theta\right.
$$$$
-2 V_{1, \theta}+4 U_{\psi 1, \theta \psi}+4 U_{\phi 1, \theta \phi}+4 C_{12, u} \cot \theta-8 C_{12, u} \tan \theta-4 C_{22, u} \cot \theta-4 C_{22, u} \tan \theta
$$$$
\left.-14 U_{\theta 2, u}+4 D_{22, u} \sec \theta+4 D_{12, u} \csc \theta+4 C_{12, u}\right)+O\left(r^{-4}\right)=0 \text {. }
$$

$R_{u \phi}=0$ :

$\frac{1}{r^{3 / 2}}\left(4 D_{11, u} \cos \theta-2 D_{11, u} \sin \theta \tan \theta-5 U_{\phi 1, u} \sin ^{2} \theta+2 D_{31, u \psi} \tan \theta+2 C_{21, u \phi}+2 D_{11, u \theta} \sin \theta\right)$

$+\frac{1}{8 r^{5 / 2}}\left(15 U_{\phi 1, \psi \psi} \sin ^{2} \theta-4 U_{\phi 1, \psi \psi} \tan ^{2} \theta-4 U_{\theta 1, \phi} \cot \theta-4 U_{\theta 1, \phi} \tan \theta-2 V_{1, \phi}+4 U_{\psi 1, \phi \psi}\right.$

$-12 U_{\phi 1, \theta} \cos \theta \sin \theta+4 U_{\phi 3, \theta} \sin ^{2} \theta \tan \theta+4 U_{\theta 1, \theta \phi}-4 U_{\phi 3, \theta \theta} \sin ^{2} \theta+D_{12, u \theta} \sin \theta$

$\left.-4 D_{12, u} \sin \theta \tan \theta-14 U_{\phi 2, u} \sin ^{2} \theta+4 D_{32, u \psi} \tan \theta+4 C_{22, u \phi}+4 D_{12, u \theta} \sin \theta\right)+O\left(r^{-3}\right)=0$.

$R_{u \psi}=0$ :

$$
\begin{aligned}
\frac{1}{4 r^{3 / 2}} & \left(2 D_{21, u} \cos \theta \cot \theta-4 D_{21, u} \sin \theta-5 U_{\psi 1, u} \cos ^{2} \theta-2 C_{11, u \psi}-2 C_{21, u \psi}+2 D_{31, u \phi} \cot \theta\right. \\
& \left.+2 D_{21, u \theta} \cos \theta\right)+\frac{1}{8 r^{5 / 2}}\left(15 U_{\psi 1} \cos ^{2} \theta+4 U_{\theta 1, \psi} \cot \theta+4 U_{\theta 1, \psi} \tan \theta-2 V_{1, \psi}+4 U_{\phi 3, \phi \psi}\right. \\
& -4 U_{\psi 1, \phi \phi} \cot ^{2} \theta-4 U_{\psi 1, \psi} \cos ^{2} \theta \cot \theta+12 U_{\psi 1, \theta} \cos \theta \sin \theta+4 U_{\theta 1, \theta \psi}-8 D_{22, u} \sin \theta \\
& \left.-14 U_{\psi 2, u} \cos ^{2} \theta-4 C_{12, u \psi}-4 C_{22, u \psi}+4 D_{32, u \phi} \cot \theta+4 D_{22, u \theta} \cos \theta\right)+O\left(r^{-3}\right)=0 .
\end{aligned}
$$

\section{APPENDIX B: DERIVATION FOR EQUATIONS (58) and (59)}

Here we sketch the derivation for Eqs. (58) and (59). We begin with

$$
\mathcal{D}_{B} \mathcal{D}_{A} F=\mathcal{D}_{B} \mathcal{D}_{A} \mathcal{D}_{C} f^{C}=\mathcal{D}_{B}\left(\mathcal{D}_{C} \mathcal{D}_{A} f^{C}-{ }^{(3)} R_{A C} f^{C}\right)=-\mathcal{D}_{B} \mathcal{D}_{C} \mathcal{D}^{C} f_{A}-2 \mathcal{D}_{A} \mathcal{D}_{B} \partial_{u} f-2 \mathcal{D}_{B} f_{A},
$$

where ${ }^{(3)} R_{A B}$ is the Ricci tensor with respect to $h_{A B}^{(0)}$. In the second and last line, we used the definition of Riemann tensor and Eq. (55), respectively. We also used the fact that that Riemann tensor with respect to $h_{A B}^{(0)}$ is ${ }^{(3)} R_{A B C D}=h_{A C}^{(0)} h_{B D}^{(0)}-h_{A D}^{(0)} h_{B C}^{(0)}$. Using the definition of Riemann tensor twice, the first term in the last line of the right-hand side becomes 
$\mathcal{D}_{B} \mathcal{D}_{C} \mathcal{D}^{C} f_{A}=\mathcal{D}_{C} \mathcal{D}_{B} \mathcal{D}^{C} f_{A}-2 \mathcal{D}_{B} f_{A}+h_{A B}^{(0)} \mathcal{D}_{C} f^{C}-\mathcal{D}_{A} f_{B}=\mathcal{D}^{2} \mathcal{D}_{B} f_{A}+2 h_{A B}^{(0)} \mathcal{D}_{C} f^{C}-2\left(\mathcal{D}_{A} f_{B}+\mathcal{D}_{B} f_{A}\right)$

Substituting this into Eq. (B1) and using the symmetry of indices $A$ and $B$, we have

$$
\mathcal{D}_{B} \mathcal{D}_{A} F=\frac{1}{2}\left[-\mathcal{D}^{2}\left(\mathcal{D}_{A} f_{B}+\mathcal{D}_{B} f_{A}\right)-4 h_{A B}^{(0)} F+2\left(\mathcal{D}_{A} f_{B}+\mathcal{D}_{B} f_{A}\right)-4 \mathcal{D}_{A} \mathcal{D}_{B} \partial_{u} f\right]
$$

Then, using Eq. (55) and $f_{, u}=-(1 / 3) F$, we obtain

$$
\mathcal{D}_{B} \mathcal{D}_{A} F=-\frac{1}{3} h_{A B}^{(0)} \mathcal{D}^{2} F-\frac{4}{3} h_{A B}^{(0)} F+\frac{2}{3} \mathcal{D}_{A} \mathcal{D}_{B} F
$$

The trace part implies that

$$
\mathcal{D}^{2} F+3 F=0
$$

Using this, Eq. (B4) becomes

$$
\mathcal{D}_{A} \mathcal{D}_{B} F=\frac{1}{3} \mathcal{D}^{2} F h_{A B}^{(0)} \text {. }
$$

${ }^{1}$ R. Penrose, Phys. Rev. Lett. 10, 66 (1963).

${ }^{2}$ R. M. Wald, General Relativity (University of Chicago Press, Chicago, 1984).

${ }^{3}$ T. Shiromizu and S. Tomizawa, Phys. Rev. D 69, 104012 (2004); e-print arXiv:gr-qc/0401006

${ }^{4}$ K. Tanabe, N. Tanahashi, and T. Shiromizu, e-print arXiv:0902.1583.

${ }^{5}$ S. Hollands and A. Ishibashi, J. Math. Phys. 46, 022503 (2005); e-print arXiv:gr-qc/0304054.

${ }^{6}$ S. Hollands and R. M. Wald, Class. Quantum Grav. 21, 5139 (2004); e-print arXiv:gr-qc/0407014.

${ }^{7}$ A. Ishibashi, Class. Quantum Grav. 25, 165004 (2008); e-print arXiv:0712.4348.

${ }^{8}$ H. Bondi, M. G. J. van der Burg, and A. W. K. Metzner, Proc. R. Soc. London, Ser. A 269, 21 (1962).

${ }^{9}$ E. T. Newman and R. Penrose, J. Math. Phys. 7, 863 (1966).

${ }^{10}$ R. K. Sachs, Proc. R. Soc. London, Ser. A 270, 103 (1962).

${ }^{11}$ R. K. Sachs, Phys. Rev. 128, 2851 (1962).

${ }^{12}$ R. C. Myers and M. J. Perry, Ann. Phys. 172, 304 (1986). 